\title{
Downregulation of miR-135a predicts poor prognosis in acute myeloid leukemia and regulates leukemia progression via modulating HOXA10 expression
}

\author{
HONGWEI XU ${ }^{1}$ and QUAN WEN ${ }^{2}$ \\ ${ }^{1}$ Clinical Laboratory, The Second Affiliated Hospital of Harbin Medical University, Harbin, Heilongjiang 150086; \\ ${ }^{2}$ General Internal Medicine, The Fourth Affiliated Hospital of Harbin Medical University, \\ Harbin, Heilongjiang 150001, P.R. China
}

Received February 12, 2018; Accepted May 18, 2018

DOI: $10.3892 / \mathrm{mmr} .2018 .9066$

\begin{abstract}
MicroRNA-135a (miR-135a) has been shown to exert important roles in various human cancer types, such as glioblastoma, thyroid carcinoma and renal carcinoma. However, the function of miR-135a in acute myeloid leukemia (AML) remains largely unknown. In the present study, it was demonstrated that miR-135a expression was significantly downregulated in AML cells compared with normal control cells. Furthermore, the downregulation of miR-135a in patients with AML predicted poor prognosis. Through functional experiments, overexpression of miR-135a was demonstrated to significantly inhibit the proliferation and cell cycle of AML cells, while it promoted cellular apoptosis. miR-135a directly targeted HOXA10 in AML cells. miR-135a overexpression significantly suppressed the mRNA and protein levels of HOXA10 in AML cells. Moreover, there was an inverse association between miR-135a expression and HOXA10 level in AML samples. Additionally, by ectopic expression of HOXA10, restoration of HOXA10 significantly abolished the effects of miR-135a overexpression on AML cell proliferation, cell cycle and apoptosis. In conclusion, the present study demonstrated that miR-135a serves as a tumor suppressor in AML by targeting HOXA10, and miR-135a may be a promising prognostic biomarker for AML patients.
\end{abstract}

\section{Introduction}

Acute myeloid leukemia (AML) is one kind of hematological malignant cancers and characterized by an incensement of undifferentiated myeloid progenitor cells in peripheral blood

Correspondence to: Professor Hongwei Xu, Clinical Laboratory, The Second Affiliated Hospital of Harbin Medical University, 248 Xuefu Road, Nangang, Harbin, Heilongjiang 150086, P.R. China E-mail: hongweixux@163.com

Key words: miR-135a, HOXA10, acute myeloid leukemia, proliferation, apoptosis and bone marrow $(1,2)$. AML cells were clinically heterogeneous and their progenitor cells have a self-renewal potential to generate the bulk of leukemia cells $(3,4)$. The incidence and mortality are gradually increasing in developing countries (5). In China, the incidence of AML is the highest among young patients (5). Chemotherapy consisting paclitaxel, cisplatin or others is the main approach for AML treatment (6). However, because of therapy resistance, tumor metastasis and recurrence, the outcomes of AML patients remain very poor (7). AML has become a great problem for public health. Thus, it is critical to elucidate the underlying molecular mechanism of AML development and progression, which will contribute to the development of effective therapeutic strategies for AML.

MicroRNAs (miRNAs/miRs) are a class of small noncoding RNAs with a length of about 22 nucleotides and expressed in nearly all kinds of cells and tissues (8). miRNAs have been demonstrated to regulate gene expression post-transcriptionally via specifically binding to the 3'-UTR region of target mRNAs for degradation $(9,10)$. In the past decades, a vast of evidence indicates that miRNAs exert vital functions in various biological processes, such as cell proliferation, apoptosis, migration and invasion (11). There was a close relationship between miRNA expression and human cancers, including lung cancer (12), bladder cancer (13), hepatocellular carcinoma (14) and AML (15). miRNAs could regulate the development and progression of cancers through serving as oncogenes or tumor suppressors (16). Aberrant expression of miRNAs is often observed in human cancer. Therefore, it is important to define the function and mechanism of miRNAs involved in cancers.

miR-135a has been reported to serve as tumor suppressor or an oncogene in different cancers. However, the role of miR-135a in AML remains largely unknown. In the present study, we found that miR-135a was downregulated in AML samples and its low expression predicted poor prognosis. Furthermore, we found that miR-135a overexpression inhibited the proliferation and induced apoptosis of AML cells. miR-135a could arrest AML cell cycle progression. In mechanism, we showed that miR-135a directly targeted HOXA10, whose restoration rescued the effects of miR-135a mimic transfection in AML cells. Taken together, our study identified miR-135a as a tumor 
suppressor in AML by targeting HOXA10 and a prognostic marker for AML patients.

\section{Materials and methods}

Clinical specimens. All the blood samples from AML patients $(n=29)$ and the normal volunteers $(n=11)$ were obtained from the Second Affiliated Hospital of Harbin Medical University (Harbin, China). All the patients were diagnosed as AML according to the pathological and clinic features. The normal volunteers were the healthy persons without any diseases. Informed consent was obtained from each patient, and the research protocols were approved by the Ethics Committee of the Second Affiliated Hospital of Harbin Medical University (Harbin, China).

Cell culture and transfection. AML cell lines including HL60, AML193, AML2, AML5 and HS-5 normal cells from marrow stroma were primarily purchased from ATCC and maintained in Eagle's Minimum Essential Medium, supplemented with $10 \%$ fetal bovine serum (both from Gibco; Thermo Fisher Scientific, Inc.) and $1 \%$ penicillin/streptomycin (Invitrogen; Thermo Fisher Scientific, Inc.) at $37^{\circ} \mathrm{C}, 5 \% \mathrm{CO}_{2}$. Cell transfection was conducted using Lippofectamine 2000 (Invitrogen; Thermo Fisher Scientific, Inc.) according to the manufacturer's protocol.

The coding sequence of HOXA10 was amplified from human cDNA and inserted into pcDNA6.1 (Invitrogen; Thermo Fisher Scientific, Inc.) vectors. miR-135a mimic (5'-AGUGUA UCCUUAUUUUUCGGUAU-3'), miR-135a inhibitor (5'-UCA CAUAGGAAUAAAAAGCCAUA-3') and mimic controls (5'-AUUCUCCGAACGUGUCACGUAGU-3') were purchased from Shanghai GenePharma Co., Ltd. (Shanghai, China) and used at a final concentration of $25 \mathrm{nmol} / \mathrm{l}$. Cell transfection was performed by using Lipo2000 (Invitrogen; Thermo Fisher Scientific, Inc.) in accordance with the manufacturer's instructions.

Cell proliferation assay. AML5 and HL60 cells were transfected with miR-135a mimics or the controls in 6-well plates for $24 \mathrm{~h}$ and then collected to seed in 96-well plates incubating for 1,3 and 5 days. $10 \mu \mathrm{l} \mathrm{CCK-8}$ reagents were added to cultures and incubated for $2 \mathrm{~h}$; measurement of absorbance of each well at $450 \mathrm{~nm}$ using a SUNRISE Microplate Reader (Tecan Group, Ltd., Mannedorf, Switzerland) was then performed.

Reverse transcription and quantitative PCR. Peripheral blood mononuclear cells from AML patients or normal individuals were separated using Ficoll Hypaque ${ }^{\circledR}$ (Sigma-Aldrich; Merck KGaA, Darmstadt, Germany) gradient centrifugation, and total RNAs were extracted using TRIzol Reagent (TransStart, Beijing, China) following the manufacturer's instructions. The first strand cDNA was compounded using a Tianscript RT kit (Tiangen Biotech, Beijing, China). PCR amplification was performed using TaqMan Human MicroRNA Assay (Applied Biosystems; Thermo Fisher Scientific, Inc.) and UltraSYBR Mixture (CW0957; CWBio, Beijing, China) in LC 480 PCR System (Roche Diagnostics, Indianapolis, IN, USA). U6 and $18 \mathrm{~S}$ was employed as reference genes to normalize the expression of miR-135a or HOXA10. Relative expression level was analyzed on the basis of the $2^{-\Delta \Delta \mathrm{Cq}}$ method (17). The primers used were synthesized and purchased from Genecopoeia (Guangzhou, China). The primer sequences were as follows: universal miRNA qRT-PCR primer (5'-AACGAGACGACG ACAGAC-3'), U6 (5'-GCAAATTCGTGAAGCGTTCCA TA-3'), miR-135a (5'-TATGGCTTTTTATTCCTATGT-3'), HOXA10 (forward, 5'-ATCTGCTCCCTTCGCCAAAT-3' and reverse, 5'-CTGATGAGCGAGTCGACCAA-3') and 18S (forward, 5'-ATCAAAACCAACCCGGTCAG-3' and reverse, 5'-CCCCGTCACCCGTGGTCACCA-3').

Cell cycle and apoptosis assay. To determine the effect of miR-135a on cell apoptosis, AML5 and HL60 cells were transfected with miR-135a mimics or miR-NC for $36 \mathrm{~h}$, then cells were harvested, washed with PBS, and centrifuged at $500 \mathrm{~g}$ at $4^{\circ} \mathrm{C}$ for $10 \mathrm{~min}$. Then cells were incubated with Annexin $\mathrm{V}$ in the dark for $15 \mathrm{~min}$, propidium iodide (PI) was added $5 \mathrm{~min}$ before the end of incubation. After centrifugation for $5 \mathrm{~min}$, cells were resuspended using PBS and FACS was performed on a FACSCalibur flow cytometer (BD Biosciences, Franklin Lakes, NJ, USA). The effect of miR-135a on cell cycle was further assessed as well. After transfection, cells were washed with PBS, fixed with $75 \%$ ethanol for $1 \mathrm{~h}$ at $-20^{\circ} \mathrm{C}$. After washing with PBS, cells were incubated in PI solution for $20 \mathrm{~min}$ at RT. Then cells were analyzed with fluorescence-activated cell sorting (FACS).

Western blot analysis. Proteins were extracted from whole cell lysates and separated by sodium dodecyl sulfate-polyacrylamide gel electrophoresis, then transferred to a polyvinylidene fluoride (PVDF) membrane. The following primary antibodies were used: anti-cyclin D1 (1:1,000; cat. no. 2978) and anti-cyclin E1 (1:1,000, cat. no. 20808; both from Cell Signaling Technology, Inc., Danvers, MA, USA), anti-HOXA10 (1:1,000, cat. no. SAB2101061; Sigma-Aldrich; Merck KGaA), anti-PCNA $(1: 3,000$; cat. no. 13110) and mouse anti-GAPDH (1:5,000; cat. no. 5174; both from Cell Signaling Technology, Inc.). Membranes were incubated with primary antibodies at $4^{\circ} \mathrm{C}$ overnight, followed by incubation with the horseradish peroxidase-conjugated secondary anti-bodies (1:5,000; Abcam, Cambridge, MA, USA) for $1 \mathrm{~h}$ at $25^{\circ} \mathrm{C}$. Ultimately, the membrane was treated with chemiluminescence reagents (Santa Cruz Biotechnology, Inc., Dallas, TX, USA) according to the manufacturer's instructions, and the image was analyzed.

Luciferase reporter assay. The potential miRNA binding sites of miR-135a predicted by computer-aided algorithms were obtained from microRNA.org target program (www. microRNA.org). The wild-type 3'-UTR of HOXA10 predicted to interact with miR-135a, together with mutant binding sequence within the predicted target sites, were synthesized by Shanghai GenePharma Co., Ltd., inserted into the pmirGLO Dual-Luciferase vector (Promega Corporation, Madison, WI, USA). AML cells were cotransfected with $500 \mathrm{ng}$ of the luciferase construct along with miR-135a and miR-135a control using Lipofectamine ${ }^{\mathrm{TM}} 2000$ reagent, in accordance with the manufacturer's protocol. Following cultivation for $48 \mathrm{~h}$, the transfected cells were collected and evaluated for luciferase activity using a Dual Luciferase Reporter Assay kit (Promega Corporation) in accordance with the manufacturer's manual. The activity of firefly luciferase was normalized to 

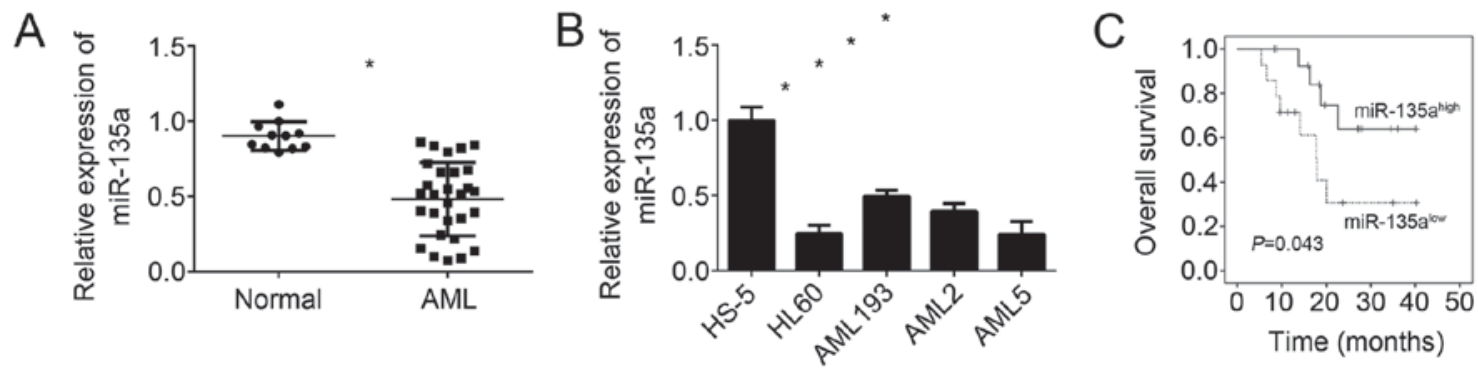

Figure 1. miR-135a was downregulated in AML. (A) miR-135a expression in blood samples from AML patients and the normal ones was determined by real time RT-PCR. Blood cells were isolated from AML patients and the normal ones, and RNAs were extracted. (B) Relative expression of miR-135a in AML cell lines was measured by qRT-PCR. (C) Correlation between miR-135a expression levels with overall survival in AML patients. The mean value of miR-135a was chosen as the cut-off. The results represented three independent experiments and were expressed as mean $\pm \mathrm{SD}$. " $\mathrm{P}<0.05$ vs. the control group.
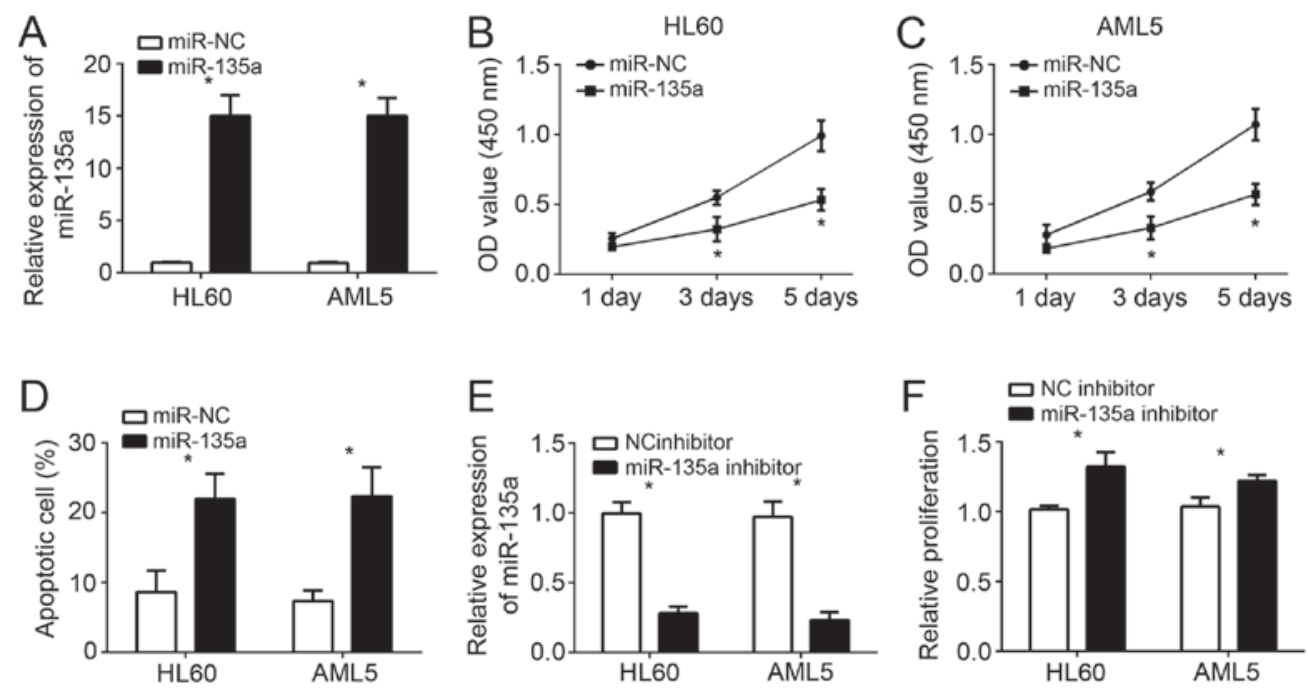

Figure 2. miR-135a inhibited cell proliferation and induced apoptosis in AML. (A) qRT-PCR analysis indicated miR-135a was significantly upregulated in HL60 and AML5 cells. CCK-8 assays were used for detection of cell proliferation in (B) HL60 and (C) AML5 cells transfected with miR-135a mimics or controls. (D) Cell apoptosis was determined by fluorescence-activated cell sorting in HL60 and AML5 cells stained with Annexin V/PI. (E) Relative expression of miR-135a in HL60 and AML5 cells by qRT-PCR. (F) CCK-8 assays were performed to evaluate cell proliferation. Cellular viability was determined $72 \mathrm{~h}$ after transfection. The results represented three independent experiments and were expressed as mean \pm SD. "P $<0.05$ vs. the control group.

that of Renilla luciferase. The results were obtained from three independent experiments performed in duplicate.

Statistical analysis. Each experiment was repeated at least three times. All data are expressed in terms of means \pm the SD. The Kaplan-Meier method was used to calculate the survival curve, and log-rank test to determine statistical significance. The differences between groups were analyzed using two-tail Student's t-test or one-way ANOVA followed by Tukey's post hoc test. $\mathrm{P}<.05$ was considered statistically significant.

\section{Results}

miR-135a is downregulated in AML. In order to explore the cellular functions of miR-135a in AML, we firstly determined the expression of miR-135a in blood samples with AML by qRT-PCR. We found that the expression of miR-135a was significantly downregulated in AML samples compared to normal samples (Fig. 1A). Besides, miR-135a levels in AML cell lines were also lower than in normal cell line (HS-5) (Fig. 1B). Then we divided these AML samples into two subgroups base on miR-135a levels and performed Kaplan-Meier curve analysis.
We found that higher expression of miR-135a in AML patients predicted better prognosis (Fig. 1C). These results suggested that miR-135a may exert a role in human AML progression and serve as a predictor for patients' prognosis.

miR-135a inhibits cell proliferation and induces apoptosis in AML. From Fig. 1B, we knew that miR-135a levels were relative lower in HL60 and AML5 cells than other cell lines. Therefore, we chose these two cell lines for further analysis. We overexpressed miR-135a in HL60 and AML5 cells through transfection with miR-135a mimics. As shown by qRT-PCR analysis, the levels of miR-135a were significantly upregulated in HL60 and AML5 cells (Fig. 2A). CCK-8 assays were used to examine the cellular proliferation. The results showed that overexpression of miR-135a significantly suppressed the cell growth in HL60 and AML5 cells at day 3 and 5 (Fig. 2B and C). Furthermore, we performed fluorescence activated cell sorter (FACS) analysis to check cell apoptosis. We found that miR-135a overexpression markedly elevated the percentages of apoptotic cells (Fig. 2D). To further confirm it, we inhibited miR-135a by transfection with miR-135a inhibitors in AML cells (Fig. 2E). CCK-8 assays indicated that miR-135a 

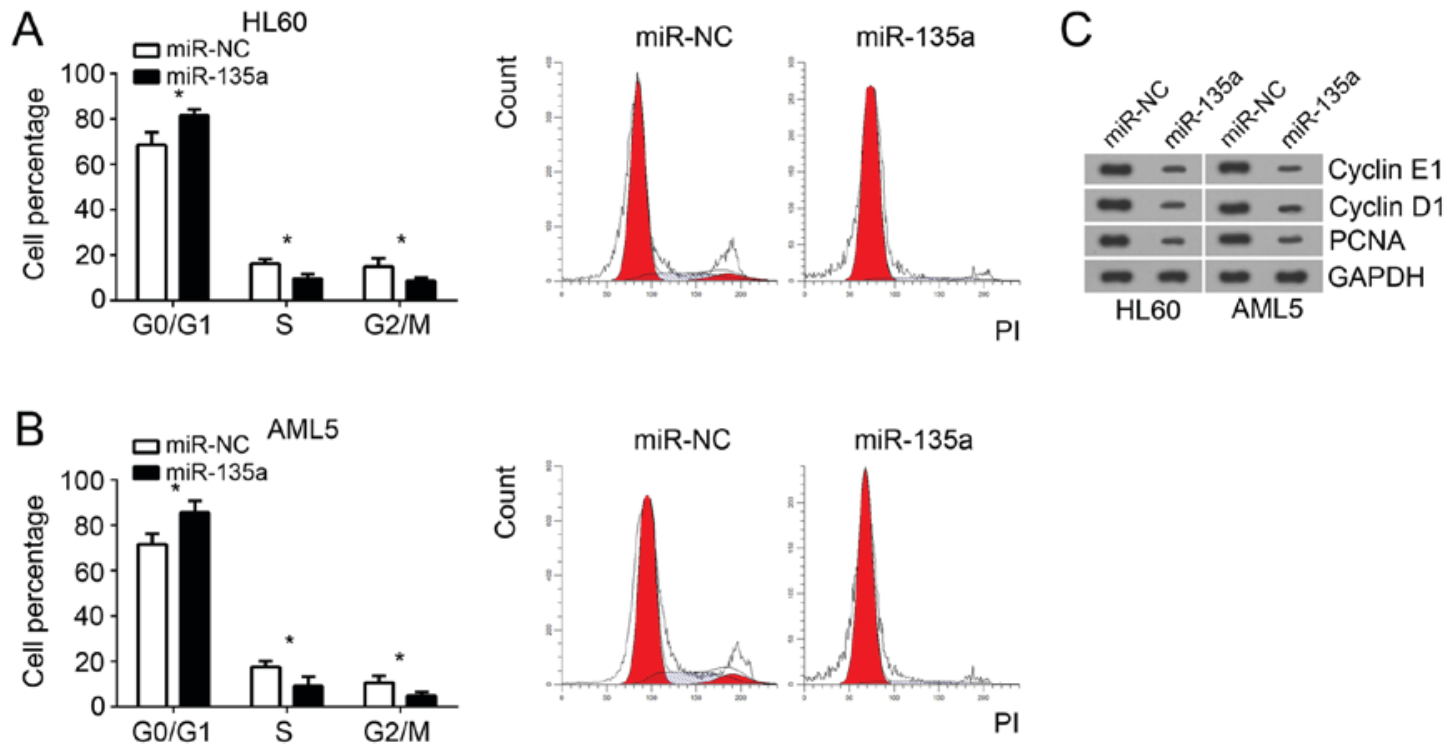

Figure 3. miR-135a suppressed cell cycle progression. Cell cycle distribution was determined by fluorescence-activated cell sorting in (A) HL60 and (B) AML5 cells transfected with miR-135a mimics or controls. (C) Cell cycle-related protein levels were measured by western blot. The results represented three independent experiments and were expressed as mean $\pm \mathrm{SD}$. ${ }^{*} \mathrm{P}<0.05$ vs. the control group.

A

5'-GGGAAAAAGCCAUA-3' HOXA10 3'UTR

3'-UUAUUUUUCGGUAU-5' miR-135a

5'-GGGAUUUUCGGUAA-3' HOXA10 3'UTR Mut
B

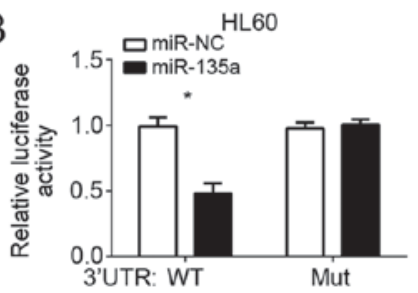

C

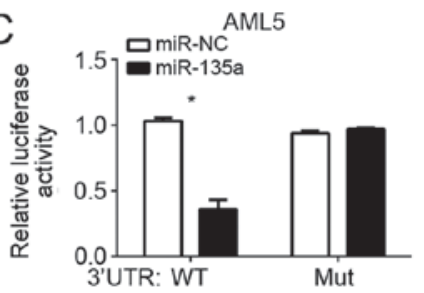

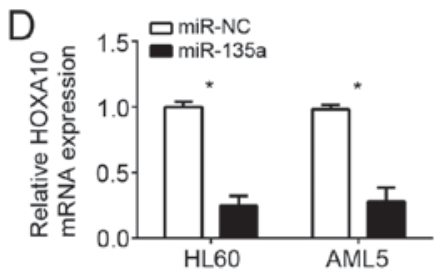

$\mathrm{E}$

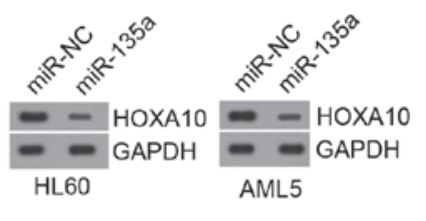

$\mathrm{F}$

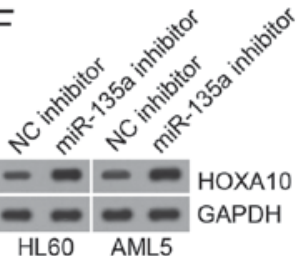

Figure 4. HOXA10 was a target of miR-135a. (A) Potential binding site of miR-135a in the 3'-UTR of HOXA10 mRNA. Luciferase reporter assays indicated that miR-135a overexpression inhibited the luciferase activity in (B) HL60 and (C) AML5 cells transfected with WT-3'-UTR, but not Mut-3'-UTR. (D) qRT-PCR was used to measure the mRNA level of HOXA10 in HL60 and AML5 cells transfected with miR-135a mimics or controls. (E) Western blot was utilized to check the protein level of HOXA10 in HL60 and AML5 cells transfected with miR-135a mimics or controls. (F) HOXA10 protein level was determined by western blot in HL60 and AML5 cells. The results represented three independent experiments and were expressed as mean \pm SD. "P $<0.05$ vs. the control group.

inhibition significantly promoted the proliferation of HL60 and AML5 cells (Fig. 2F).

miR-135a suppresses cell cycle progression. Cell proliferation was directly influenced by cell cycle. Therefore, we analyzed the effect of miR-135a on cell cycle by FACS. We found that overexpression of miR-135a significantly increased the numbers of HL60 and AML5 cells in G0/G1 phase (Fig. 3A and B). However, the HL60 and AML5 cells in S and G2/M phase were significantly decreased after miR-135a overexpression (Fig. 3A and B). Moreover, through western blot, we found that overexpression of miR-135a significantly reduced the protein levels of cyclin E1, cylcin D1 and PCNA in HL60 and AML5 cells (Fig. 3C), which indicated that miR-135a overexpression significantly inhibited cell cycle progression.
HOXA10 is a target of miR-135a. To search the target genes of miR-135a in AML cells, we used online prediction software (TargetScan 7.0 and miRBase) to look for the most possible target genes. Among all results, HOXA10 ranked top and has been reported to promote AML progression. Therefore, we chose them for following validation. There was a potential binding site of miR-135a in the 3'-UTR region of HOXA10 mRNA (Fig.4A). Then we performed luciferase reporter assays. Results showed that the luciferase activity of WT-3'-UTR HOXA10 in HL60 and AML5 cells was significantly downregulated by overexpression of miR-135a while no change was observed in the mutated 3'UTR transfection (Fig. 4B and C). Furthermore, qRT-PCR and western blot analysis indicated that overexpression of miR-135a significantly downregulated the mRNA and protein levels of HOXA10 in HL60 and AML5 

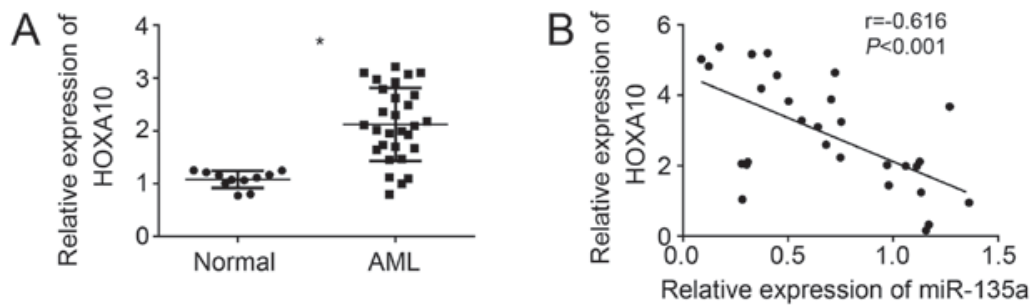

Figure 5. miR-135a was negatively related to HOXA10 expression in AML. (A) Relative expression of HOXA10 in AML samples and normal samples was examined by qRT-PCR. (B) Relationship of HOXA10 mRNA and miR-135ap expression in AML samples. The results represented three independent experiments and were expressed as mean $\pm \mathrm{SD}$. ${ }^{*} \mathrm{P}<0.05$ vs. the control group.
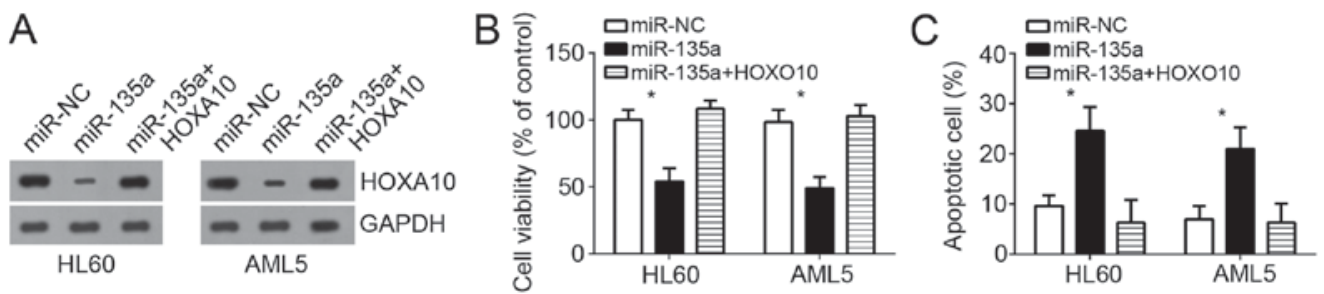

Figure 6. Restoration of HOXA10 reversed the effects of miR-135a overexpression. (A) Western blot was used to check the protein levels of HOXA10. (B) CCK-8 assays were used to measure the cellular proliferation. (C) Cell apoptosis was determined by FACS. The results represented three independent experiments and were expressed as mean $\pm \mathrm{SD}$. ${ }^{*} \mathrm{P}<0.05$ vs. the control group.

cells (Fig. 4D and E), whereas miR-135a knockdown promoted HOXA10 expression (Fig. 4F).

miR-135a is negatively associated with HOXA10 expression in $A M L$. To further confirm the relationship of miR-135a and HOXA10 in AML cells, we analyzed the expression patterns of HOXA10 in AML sample cells by qRT-PCR. We found that HOXA10 expression was upreuglated in AML sample cells compared to normal cells (Fig. 5A). Moreover, we found that HOXA10 mRNA level was negatively correlated with miR-135a expression in AML samples (Fig. 5B). These data further confirmed that miR-135a directly targeted HOXA10 in AML cells.

Restoration of HOXA10 reversed the effects of miR-135a overexpression. We have demonstrated HOXA10 was a direct target of miR-135a in AML cells. In order to determine whether miR-135a suppressed the proliferation and promoted apoptosis of AML cells through HOXA10, HL60 and AML5 cells were transduced with HOXA10. Western blot analysis results indicated that HOXA10 expression was significantly upregulated in HL60 and AML5 cells (Fig. 6A). Then cell survival ability was examined by CCK-8 assays in HL60 and AML5 cells. Results indicated that miR-135a overexpression decreased the survival ability and promoted cell apoptosis in HL60 and AML5 cells while restoration of HOXA10 significantly abrogated these effects of miR-135a overexpression (Fig. 6B and C). Taken together, our data demonstrated that miR-135a suppressed AML cell proliferation and induced apoptosis through directly targeting HOXA10.

\section{Discussion}

AML is one the most malignant cancers and the pathogenesis of AML need to be elucidated. miRNAs have been reported to participate in the development and progression of almost all kinds of human cancers, including breast cancer (18), cervical cancer (19), gastric cancer (20) and AML (15). Therefore, elucidating the mechanism of miRNA function will be crucial for the development of therapeutic strategies. Several miRNAs have been demonstrated to exert important roles on AML progression. For example, Wan et al reported that miR-103 confers the resistance to long-treatment of adriamycin to human leukemia cells by regulation of COP1 (21). Liu et al showed that miR-34a induced apoptosis and suppressed autophagy through targeting HMGB1 in AML cells (22). Wang et al reported that upregulation of miR-125b suppresses the invasion and proliferation of human AML cells, and induced cellular apoptosis through regulating NF- $\kappa \mathrm{B}$ pathway (23). Ke et al showed that miR-192 suppressed the proliferation, cell cycle transition of AML cells by targeting CCNT2 (24). In addition, Wang et al demonstrated that miR-183 enhanced the proliferation of pediatric AML cells by targeting programmed cell death 6 (25). miR-135a has been shown to aberrantly expressed in several cancer, including non-small cell lung cancer (26), thyroid carcinoma (27), glioblastoma (28), pancreatic cancer (29), hepatocellular carcinoma (30) and gastric cancer (31). However, the function of miR-135a in AML remains elusive. In this study, we showed that miR-135a expression was significantly downregulated in AML samples and cell lines. Moreover, the expression levels of miR-135a were correlated with the prognosis of AML patients. Furthermore, we demonstrated that miR-135a overexpression suppressed the proliferation and induced apoptosis of AML cells by targeting HOXA10.

HOXA10 belongs to the HOX gene superfamily encoding a class of transcription factors that regulate cell proliferation, differentiation and migration $(32,33)$. Dysregulation of HOXA10 has been observed in several cancers, including 
ovarian cancer $(34,35)$, colorectal cancer (36), oral squamous cell carcinoma (37), hepatocellular carcinoma (38), prostate carcinoma (39) and pancreatic cancer (40). As an oncogene, HOXA10 has also been demonstrated to promote the progression of human AML (41). In addition, Wang et al reported that constitutively active SHP2 cooperates with Hox A10 overexpression to induce AML (42). However, how HOXA10 expression was regulated by miRNAs in AML cells remains largely unknown. In our study, we found that HOXA10 was a direct target of miR-135a in AML cells. We showed that overexpression of miR-135a suppressed the luciferase activity of HOXA10 3'UTR in HL60 and AML5 cells. And miR-135a overexpression significantly reduced the mRNA and protein levels of HOXA10 in HL60 and AML5 cells. Furthermore, we also showed that HOXA10 expression was upregulated and negatively correlated with that of miR-135a in AML sample. Then through functional experiments, we found that overexpression of HOXA10 significantly reversed the effects of miR-135a transfection on AML cell proliferation and apoptosis. These results indicated that HOXA10 serves as an oncogene and is critical for the function of miR-135a in AML.

In conclusion, our study elucidated the essential role of miR-135a in AML progression and demonstrated its functional mechanism that miR-135a suppressed AML proliferation and induced apoptosis of AML cell through targeting HOXA10. Our study indicated that HOXA10 could act as a prognostic predictor for AML patients and might be a promising therapeutic target for AML treatment.

\section{Acknowledgements}

Not applicable.

\section{Funding}

No funding was received.

\section{Availability of data and materials}

All data generated or analyzed during this study are included in this published article.

\section{Authors' contributions}

HX initiated, designed this work, analyzed, interpreted the results and wrote this manuscript. QW performed the experiments. All authors read and approved the final manuscript.

\section{Ethics approval and consent to participate}

For the use of human samples, the protocol for this study was approved by the Institutional Ethics Committee of The Second Affiliated Hospital of Harbin Medical University and all enrolled patients signed a written informed consent document.

\section{Consent for publication}

All patients within this study provide consent for the publication of their data.

\section{Competing interests}

The authors declare that they have no competing interests.

\section{References}

1. Estey EH: Acute myeloid leukemia: 2013 update on riskstratification and management. Am J Hematol 88: 318-327, 2013.

2. Passegue E, Jamieson CH, Ailles LE and Weissman IL: Normal and leukemic hematopoiesis: Are leukemias a stem cell disorder or a reacquisition of stem cell characteristics? Proc Natl Acad Sci USA 100 (Suppl 1): S11842-S11849, 2003.

3. Eppert K, Takenaka K, Lechman ER, Waldron L, Nilsson B, van Galen P, Metzeler KH, Poeppl A, Ling V, Beyene J, et al: Stem cell gene expression programs influence clinical outcome in human leukemia. Nat Med 17: 1086-1093, 2011.

4. Kreso A and Dick JE: Evolution of the cancer stem cell model. Cell Stem Cell 14: 275-291, 2014

5. Xu XH, Zhang L, Cao XX, Li J, Zhang W, Zhu TN, Cai HC, Chen M, Han X, Yang C, et al: Evaluation of the implementation rate of primary antifungal prophylaxis and the prognosis of invasive fungal disease in acute leukemia patients in China. J Infect Chemother 23: 360-367, 2017.

6. Marcucci G, Mrozek K, Radmacher MD, Garzon R and Bloomfield CD: The prognostic and functional role of microRNAs in acute myeloid leukemia. Blood 117: 1121-1129, 2011.

7. Khalaj M, Tavakkoli M, Stranahan AW and Park CY: Pathogenic microRNA's in myeloid malignancies. Front Genet 5: 361, 2014.

8. Bartel DP: MicroRNAs: Genomics, biogenesis, mechanism and function. Cell 116: 281-297, 2004.

9. Liao Q, Wang B, Li X and Jiang G: miRNAs in acute myeloid leukemia. Oncotarget 8: 3666-3682, 2017.

10. Zavala-Yoe R, Ramirez-Mendoza RA and Cordero LM: Entropy measures to study and model long term simultaneous evolution of children in Doose and Lennox-Gastaut syndromes. J Integr Neurosci 15: 205-221, 2016.

11. $\mathrm{Bu} \mathrm{W}$ and Luo T: miR-1297 promotes cell proliferation of non-small cell lung cancer cells: Involving in PTEN/Akt/Skp2 signaling pathway. DNA Cell Biol 36: 976-982, 2017.

12. Kang M, Li Y, Zhao Y, He S and Shi J: miR-33a inhibits cell proliferation and invasion by targeting CAND1 in lung cancer. Clin Transl Oncol 20: 457-466, 2018.

13. Xiong Y, Wang L, Li Y, Chen M, He W and Qi L: The long non-coding RNA XIST interacted with MiR-124 to modulate bladder cancer growth, invasion and migration by targeting androgen receptor (AR). Cell Physiol Biochem 43: 405-418, 2017.

14. Tran DDH, Kessler C, Niehus SE, Mahnkopf M, Koch A and Tamura T: Myc target gene, long intergenic noncoding RNA, Linc 00176 in hepatocellular carcinoma regulates cell cycle and cell survival by titrating tumor suppressor microRNAs. Oncogene 37: $75-85,2018$.

15. Zhang S, Zhang Q, Shi G and Yin J: MiR-182-5p regulates BCL2L12 and BCL2 expression in acute myeloid leukemia as a potential therapeutic target. Biomed Pharmacother 97: 1189-1194, 2018.

16. Zhang TJ, Lin J, Zhou JD, Li XX, Zhang W, Guo H, Xu ZJ, Yan Y, Ma JC and Qian J: High bone marrow miR-19b level predicts poor prognosis and disease recurrence in de novo acute myeloid leukemia. Gene 640: 79-85, 2018.

17. Livak KJ and Schmittgen TD: Analysis of relative gene expression data using real-time quantitative PCR and the 2(-Delta Delta C(T)) method. Methods 25: 402-408, 2001.

18. Ye P, Shi Y, An N, Zhou Q, Guo J and Long X: miR-145 overexpression triggers alteration of the whole transcriptome and inhibits breast cancer development. Biomed Pharmacother 100: 72-82, 2018.

19. Liu YL, Wang GQ, Cui HX, Li XX, Xu ZL and Wang XY: miRNA211 induces apoptosis of cervical cancer SiHa cells via down-regulation of inhibitor of apoptosis proteins. Eur Rev Med Pharmacol Sci 22: 336-342, 2018.

20. Cao Y, Song J, Ge J, Song Z, Chen J and Wu C: MicroRNA-100 suppresses human gastric cancer cell proliferation by targeting CXCR7. Oncol Lett 15: 453-458, 2018.

21. Wan L, Tian Y, Zhang R, Peng Z, Sun J and Zhang W: MicroRNA-103 confers the resistance to long-treatment of adriamycin to human leukemia cells by regulation of COP1. J Cell Biochem 119: 3846-3852, 2018. 
22. Liu L, Ren W and Chen K: MiR-34a promotes apoptosis and inhibits autophagy by targeting HMGB1 in acute myeloid leukemia cells. Cell Physiol Biochem 41: 1981-1992, 2017.

23. Wang Y, Tang P, Chen Y, Chen J, Ma R and Sun L: Overexpression of microRNA-125b inhibits human acute myeloid leukemia cells invasion, proliferation and promotes cells apoptosis by targeting NF-kappaB signaling pathway. Biochem Biophys Res Commun 488: 60-66, 2017.

24. Ke S, Li RC, Lu J, Meng FK, Feng YK and Fang MH: MicroRNA-192 regulates cell proliferation and cell cycle transition in acute myeloid leukemia via interaction with CCNT2. Int J Hematol 106: 258-265, 2017.

25. Wang X, Zuo D, Yuan Y, Yang X, Hong Z and Zhang R: MicroRNA-183 promotes cell proliferation via regulating programmed cell death 6 in pediatric acute myeloid leukemia. J Cancer Res Clin Oncol 143: 169-180, 2017.

26. Zhang T and Wang N: miR-135a confers resistance to gefitinib in non-small cell lung cancer cells by upregulation of RAC1. Oncol Res: Jan 31, 2018 (Epub ahead of print).

27. Zhao X, Sun Z, Li H, Jiang F, Zhou J and Zhang L: MiR-135a-5p modulates biological functions of thyroid carcinoma cells via targeting VCAN 3'-UTR. Cancer Biomark 20: 207-216, 2017.

28. Gomez Zubieta DM, Hamood MA, Beydoun R, Pall AE and Kondapalli KC: MicroRNA-135a regulates NHE9 to inhibit proliferation and migration of glioblastoma cells. Cell Commun Signal 15: 55, 2017.

29. Zhang X, Gao F, Zhou L, Wang H, Shi G and Tan X: UCA1 regulates the growth and metastasis of pancreatic cancer by sponging miR-135a. Oncol Res 25: 1529-1541, 2017.

30. von Felden J, Heim D, Schulze K, Krech T, Ewald F, Nashan B, Lohse AW and Wege H: High expression of micro RNA-135A in hepatocellular carcinoma is associated with recurrence within 12 months after resection. BMC Cancer 17: 60, 2017.

31. Yan LH, Chen ZN, Li L, Chen J, Wei WE, Mo XW, Qin YZ, Lin Y and Chen JS: miR-135a promotes gastric cancer progression and resistance to oxaliplatin. Oncotarget 7: 70699-70714, 2006.

32. Yoshida H, Broaddus R, Cheng W, Xie $\mathrm{S}$ and Naora $\mathrm{H}$ : Deregulation of the HOXA10 homeobox gene in endometrial carcinoma: role in epithelial-mesenchymal transition. Cancer Res 66: 889-897, 2006.
33. Bei L, Lu Y, Bellis SL, Zhou W, Horvath E and Eklund EA: Identification of a HoxA10 activation domain necessary for transcription of the gene encoding beta 3 integrin during myeloid differentiation. J Biol Chem 282: 16846-16859, 2007.

34. Zhang HY, Li JH, Li G and Wang SR: Activation of ARK5/ miR-1181/HOXA10 axis promotes epithelial-mesenchymal transition in ovarian cancer. Oncol Rep 34: 1193-1202, 2015.

35. Tang W, Jiang Y, Mu X, Xu L, Cheng W and Wang X: MiR-135a functions as a tumor suppressor in epithelial ovarian cancer and regulates HOXA10 expression. Cell Signal 26: 1420-1426, 2014.

36. Sun S, Su C, Zhu Y, Li H, Liu N, Xu T, Sun C and Lv Y: MicroRNA-544a regulates migration and invasion in colorectal cancer cells via regulation of homeobox A10. Dig Dis Sci 61: 2535-2544, 2016.

37. Carrera M, Bitu CC, de Oliveira CE, Graner E, Manninen A, Salo T and Coletta RD: HOXA10 controls proliferation, migration and invasion in oral squamous cell carcinoma. Int J Clin Exp Pathol 8: 3613-3623, 2015.

38. Xiao ZD, Jiao CY, Huang HT, He LJ, Zhao JJ, Lu ZY and Liu LX: miR-218 modulate hepatocellular carcinoma cell proliferation through PTEN/AKT/PI3K pathway and HoxA10. Int J Clin Exp Pathol 7: 4039-4044, 2014.

39. Li B, Cao X, Weng C, Wu Y, Fang X, Zhang X and Liu G HoxA10 induces proliferation in human prostate carcinoma PC-3 cell line. Cell Biochem Biophys 70: 1363-1368, 2014.

40. Cui XP, Qin CK, Zhang ZH, Su ZX, Liu X, Wang SK and Tian XS: HOXA10 promotes cell invasion and MMP-3 expression via TGF 32 -mediated activation of the $\mathrm{p} 38$ MAPK pathway in pancreatic cancer cells. Dig Dis Sci 59: 1442-1451, 2014.

41. Shah CA, Bei L, Wang H, Platanias LC and Eklund EA: HoxA10 protein regulates transcription of gene encoding fibroblast growth factor 2 (FGF2) in myeloid cells. J Biol Chem 287: 18230-18248, 2012.

42. Wang H, Lindsey S, Konieczna I, Bei L, Horvath E, Huang W, Saberwal G and Eklund EA: Constitutively active SHP2 cooperates with HoxA10 overexpression to induce acute myeloid leukemia. J Biol Chem 284: 2549-2567, 2009. 